\title{
Short-term and long-term attentional biases to frequently encountered target features
}

\author{
Li Z. Sha ${ }^{1} \cdot$ Roger W. Remington ${ }^{2,3} \cdot$ Yuhong V. Jiang ${ }^{1,3}$
}

Published online: 24 April 2017

(C) The Psychonomic Society, Inc. 2017

\begin{abstract}
It has long been known that frequently occurring targets are attended better than infrequent ones in visual search. But does this frequency-based attentional prioritization reflect momentary or durable changes in attention? Here we observed both short-term and long-term attentional biases for visual features as a function of different types of statistical associations between the targets, distractors, and features. Participants searched for a target, a line oriented horizontally or vertically among diagonal distractors, and reported its length. In one set of experiments we manipulated the target's color probability: Targets were more often in Color 1 than in Color 2. The distractors were in other colors. Participants found Color 1 targets more quickly than Color 2 targets, but this preference disappeared immediately when the target's color became random in the subsequent testing phase. In the other set of experiments, we manipulated the diagnostic values of the two colors: Color 1 was more often a target than a distractor; Color 2 was more often a distractor than a target. Participants found Color 1 targets more quickly than Color 2 targets. Importantly, and in contrast to the first set of experiments, the featural preference was sustained in the testing phase. These results suggest that short-term and long-term attentional biases are products of different statistical information. Finding a target momentarily activates its features, inducing short-term repetition priming. Long-
\end{abstract}

Li Z. Sha

lixx3632@umn.edu

1 Department of Psychology, University of Minnesota, S504 Elliott Hall, Minneapolis, MN 55455, USA

2 School of Psychology, University of Queensland, Brisbane, Queensland, Australia

3 Center for Cognitive Sciences, University of Minnesota, Minneapolis, MN, USA term changes in attention, on the other hand, may rely on learning diagnostic features of the targets.

Keywords Selective attention · Visual search · Target's frequency effect $\cdot$ Statistical learning $\cdot$ Diagnostic features

A typical scene contains many colors, shapes, and objects. These stimuli differ in various ways that influence how likely they will be attended. Research on selective attention has typically emphasized two factors: whether the stimulus is perceptually salient and whether it matches the observer's task goals. It has long been known, however, that attention may also be tuned to frequently encountered stimuli, especially if these stimuli bear behavioral relevance. The "own-name effect" illustrates this phenomenon: One's own name is highly salient, owing to its high occurrence rates and association with oneself. More broadly, features frequently associated with a target are prioritized in processing. For example, in visual search tasks involving multiple possible targets, people more accurately detect a target that occurs more frequently (Godwin et al., 2010; Godwin, Menneer, Riggs, Cave, \& Donnelly, 2015; Hout, Walenchok, Goldinger, \& Wolfe, 2015).

Featural frequency may affect attention on multiple time scales. First, inter-trial priming exerts short-term changes of attention. When a target is found on one trial, its features are in an activated state, lowering the threshold needed for activation on subsequent trials (Maljkovic \& Nakayama, 1994). Because the more frequent feature repeats more often, short-term intertrial priming contributes to effects of the target's frequency on attention. Second, statistical learning about the target's featural frequency may induce long-term changes in attention. Observers extract the statistical regularities of the target objects, including the frequency that a target has certain features. This information allows observers to increase the priority 
weights assigned to the more probable features. Stable differences in featural frequency are expected to yield persistent changes in attention. In fact, many forms of statistical learning yield long-term, durable changes in attention (Chun \& Jiang, 2003; Gebhart, Aslin, \& Newport, 2009; Jiang, Swallow, Rosenbaum, \& Herzig, 2013; Jungé, Scholl, \& Chun, 2007; Yu \& Zhao, 2015). Short-term and long-term mechanisms of the target frequency effect provide complementary ways of adapting attentional priority to the environment. Short-term mechanisms allow people to rapidly adjust to the variable aspects of the environment on the basis of recent search history. Long-term mechanisms improve search efficiency in stable visual environments.

Experimental evidence has shown that both inter-trial priming and long-term statistical learning contribute to changes in spatial attention. Specifically, when a target frequently appears in one region of space, observers are faster at finding targets occurring there relative to elsewhere. The reaction time (RT) advantage has two components: location repetition priming, and statistical learning of the target's location probability (Jiang, Sha, \& Remington, 2015; Jiang et al., 2013; Walthew \& Gilchrist, 2006). Similarly, in the domain of featural attention, both transient and durable changes have been reported (Kruijne \& Meeter, 2015, 2016; Sha, Remington, \& Jiang, 2017). However, unlike spatial attention, in which short-term and long-term effects are both present, studies on featural attention have sometimes failed to observe any long-term effects. These findings, reviewed next, suggest that stable statistical regularities in the frequency with which target features occur are insufficient to induce durable attentional biases.

In one study, Kruijne and Meeter (2015) asked participants to perform a singleton search task in which the target was a uniquely colored object (e.g., green) among homogeneous distractors (e.g., red). On some trials the target was green, and on others it was red. In the training, "biased" phase, the target was four times as likely to be in one color as the other. In a subsequent, "neutral" phase, the target was equally likely to be in either one of the two colors. Kruijne and Meeter (2015) found that participants prioritized the high-frequency color in the biased phase. However, this effect did not persist in the subsequent neutral phase. Sha et al. (2017) extended this finding to a feature search task. Participants searched for two possible target colors (Color 1 or Color 2) among distractors in other colors. Because the distractor colors were heterogeneous, the task entailed feature search rather than singleton search. Nonetheless, an attentional bias for the more probable target color disappeared immediately in the neutral testing phase.

In contrast, when the search task involved a color-shape conjunction task, Kruijne and Meeter $(2015,2016)$ found evidence for long-term changes in attention. In this task, participants searched for either a green diamond or a red diamond among distractors that were blue diamonds or red/green/blue circles, triangles, and squares. The target was four times as likely to appear in one color as in the other in the biased phase. Participants were faster at finding the target when it appeared in the high-frequency color. Notably, this RT difference extended to the subsequent neutral phase, during which the target was equally likely to be in either color. Instructions to treat the two colors equally did not eliminate the attentional bias, nor did a one-week delay between the biased and neutral phases. These data indicate that under some circumstances, frequency differences in the target's color yield durable changes in attention.

To account for differences between singleton search and conjunction search, Kruijne and Meeter $(2015,2016)$ propose that long-term attentional biases depend on episodic retrieval of the search targets. In the conjunction search task, participants need to search for diamonds of two specific colors. These two colors are retained in episodic memory, with stronger memory traces for the more frequent color. In contrast, in the singleton search task, participants do not need to search for specific colors. Rather, search can be accomplished using a singleton-detection mode (Bacon \& Egeth, 1994), in which participants search for differences in local contrasts among the stimuli. The two specific colors fail to leave strong episodic memory traces, preventing long-term attentional biases from emerging. Long-term attentional biases do not depend on task difficulty. Kruijne and Meeter (2015) showed that only shortterm changes occurred in a difficult singleton search task, whereas long-term changes were found in an easy conjunction search task.

Though searching for specific features may be important for inducing long-term attentional biases, it does not seem sufficient. The feature search task used by Sha et al. (2017) required participants to search for specific target colors among heterogeneous distractor colors. Despite the need to maintain the target colors in memory, it failed to induce durable attentional biases for the high-frequency color. What other factors may contribute to long-term changes in featural attention?

An answer to this question comes from considering different types of statistical regularities inherent in the different search tasks. One difference between feature search and conjunction search lies in the feature's utility in differentiating targets from distractors. In the feature search task (Sha et al., 2017), the two target colors (e.g., red and green), by definition, are always targets and never distractors. They are both maximally diagnostic of an object being a target. In the conjunction search task (Kruijne \& Meeter, 2015), however, targets are defined by a combination of color and shape, so a specific color typically appears as both a target and a distractor. When a color is more often associated with the target than with a distractor, it becomes more diagnostic of the target, relative to the low-frequency color. It is possible that longterm changes of attention are sensitive to how probable a feature coincides with a target rather than with a distractor. 
Following this logic, we distinguish between two types of feature-target association: the target's featural frequency and a feature's diagnostic value. A target's featural frequency can be expressed as $P($ feature $i \mid \operatorname{target})$. Given a target, what is its probability of having feature $i$ rather than other features? This association is computed on all target objects, without considering the featural composition of distractors. Because the target's features are activated in working memory, processing of the same features should be facilitated on subsequent trials (e.g., the next five to eight trials; see Maljkovic \& Nakayama, 1994). If the target is more frequently associated with a certain feature, then targets associated with this feature should gain stronger inter-trial priming and be found faster than targets associated with other features. In contrast, a feature's diagnostic value pertains to how reliably the presence of the feature is associated with a target as opposed to a distractor. It can be expressed as $P(\operatorname{target} \mid$ feature $i$ ). Given a feature $i$, what is its probability of being associated with the target rather than with a distractor? Prioritizing a feature that is more diagnostic of the target is important because it leads to successful task completion.

In four experiments, we tested the episodic-retrieval account and the diagnostic-value account - the possibility that long-term changes of attention depend on differences in diagnostic values. We manipulated the two types of color-target association, while minimizing the need to store the colors in episodic memory. In some experiments, the two colors differed only in the target's featural frequency. In other experiments, the two colors also differed in their diagnostic values. We examined conditions under which long-term changes in attention occurred.

\section{Experiment 1}

Experiment 1 tested the possibility that without a difference in diagnostic value, differences in target's featural frequency only induce short-term changes in attention. In this experiment, participants searched for a target line in a canonical orientation (horizontal or vertical) among distractor lines in noncanonical orientations ( $45^{\circ}$ to the left or the right). Once they had found the target, they pressed a button to report whether the target was long or short (Fig. 1). All the lines had different colors. Unbeknownst to the participants, the target line was always in one of two colors, such as red or green, whereas the distractor lines were in other colors. We manipulated the target's color frequency. For clarity, we refer to the frequent target color as Color 1 and the infrequent one as Color 2. The target was Color $175 \%$ of the time, and Color 2 the other $25 \%$ of the time. We examined (1) whether participants were faster finding targets in Color 1 than Color 2 and (2) whether this preference persisted in a neutral testing phase in which the target was equally likely to be Color 1 or Color 2 .

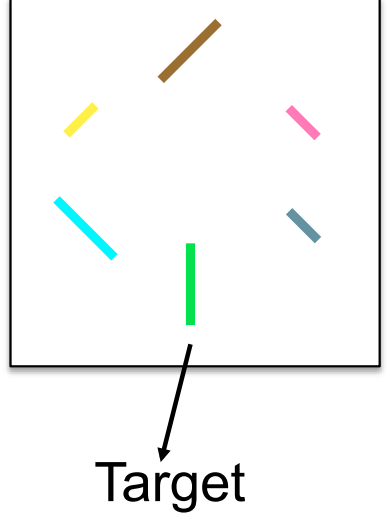

Fig. 1 Schematic illustration of a search display used in Experiment 1. Participants searched for a line in a canonical (horizontal/vertical) orientation and reported its length. Color was an incidental feature. However, the target line was in either Color 1 (75\% of the trials) or Color 2 (25\% of the trials). Color 1 and Color 2 were used exclusively for targets. The distractor lines were in various colors other than Color 1 or Color 2

In this design, both Color 1 and Color 2 always coincided with the target and never coincided with a distractor. Thus, both colors were maximally diagnostic of the target, $P(\operatorname{target} \mid$ Color 1$)=P(\operatorname{target} \mid$ Color 2$)=1$. However, the target was more often Color 1 than Color 2, $P($ Color $1 \mid$ target $)=.75>$ $P($ Color $2 \mid$ target $)=.25$. The training phase allowed us to measure the development of an RT advantage for finding targets that were associated with Color 1 . The testing phase examined the durability of this advantage.

\section{Method}

Participants The participants in this study were students from the University of Minnesota. They were 18-24 years old, had normal or corrected-to-normal visual acuity, normal color vision, and were naïve to the purpose of the study. Participants were compensated for their time with extra course credit or $\$ 10 / \mathrm{h}$.

Twelve participants (seven males, five females) with a mean age of 19.8 years completed Experiment 1.

Equipment Participants were tested individually in a room with normal interior lighting. The experiment was programmed using Psychophysics Toolbox (Brainard, 1997; Pelli, 1997) implemented in MATLAB (www.mathworks.com). Stimuli were presented on a 17-in. CRT monitor with a resolution of 1,024 $\times$ 768 pixels and a refresh rate of $75 \mathrm{~Hz}$. The viewing distance was unconstrained but was approximately $40 \mathrm{~cm}$.

Materials and stimuli Each display contained six colored lines presented at equidistant locations on an imaginary circle (Fig. 1). There were one target and five distractors. The eccentricity of each item was $5^{\circ}$. The length of each line was randomly chosen to be either $1^{\circ}$ or $2^{\circ}$. Four colors made up the 
target set: red, green, yellow, and blue. Two of these were randomly chosen as the potential target colors for a participant. These assignments were counterbalanced across participants. The two colors not chosen to be the target colors, along with six other colors generated using MATLAB's maximally distinguishable colors script (www.mathworks.com/ matlabcentral/fileexchange/29702-generate-maximallyperceptually-distinct-colors/content/distinguishable_colors. $\mathrm{m}$ ), made up the distractor set. The target's orientation was randomly assigned to be either vertical or horizontal, and the distractors' orientations were randomly assigned to be $45^{\circ}$ left-tilted or $45^{\circ}$-right-tilted (Fig. 1). All items were displayed against a black background.

Procedure Participants were asked to search for a target in a canonical orientation, specifically the single horizontal or vertical line, among tilted lines. Each trial started with a white fixation point $\left(0.5^{\circ} \times 0.5^{\circ}\right)$. After a random duration of 400 $600 \mathrm{~ms}$, the six-item search display appeared. Upon finding the target, participants reported the target's length by pressing "L" if the target was long $\left(2^{\circ}\right)$ or " $\mathrm{S}$ " if the target was short $\left(1^{\circ}\right)$. The search display remained in view until participants had responded (Fig. 1). We emphasized accuracy and speed in the task instructions and the trial feedback. Each incorrect response was followed by the computer voice speaking the sentence "That was wrong. Please try to be accurate." Correct trials were followed by a chirp, or by the text "too slow" if the RT was slower than $1,000 \mathrm{~ms}$. The next trial commenced after 1,000 ms. Participants were encouraged to take a break every 48 trials. They completed 16 blocks, with 48 trials per block.

Design We manipulated the target color's frequency. In the training phase (the first 12 blocks of trials), the target was Color 1 (high-frequency color) on $75 \%$ of the trials, and Color 2 (low-frequency color) on the remaining $25 \%$ of the trials. These two colors never appeared as distractors. In the testing phase (the last four blocks of trials), the target was associated with these two colors equally often $(50 \%$ vs. $50 \%$ ). We did not inform participants of the target frequency difference. Assignments of the high- and low-frequency colors were counterbalanced across participants. For example, if blue was the high-frequency color and yellow was the lowfrequency color for one participant, the frequency assignments for blue and yellow targets would be reversed for another participant.

Recognition We queried participants about the target's frequency at the end of the experiment. The recognition questions asked participants to estimate the percentage of trials in which the target appeared in Colors 1 and 2, respectively. Recognition questions first asked about Blocks 1-12 (the biased phase) and then about Blocks 13-16 (the neutral phase).
Similar recognition questions were used in Experiments 2, 3 , and 4. Recognition results will be reported after we have presented the search data from all four experiments.

\section{Results}

Table 1 displays the mean accuracies in all experiments, showing no evidence of a speed-accuracy trade-off (i.e., either no difference in accuracy between Color 1 and Color 2 or the difference in accuracy between the two colors was consistent with the RT results).

Incorrect trials and trials whose RT exceeded $10 \mathrm{~s}$ (fewer than $0.03 \%$ of trials were excluded as outliers in all experiments) were excluded from the RT analyses in all experiments. Figure 2 displays the mean RTs in Experiment 1.

In the training phase, a repeated measures analysis of variance (ANOVA) on the target's color (Color 1 or Color 2) and block (Blocks 1-12) showed that RTs were significantly faster for targets in Color 1 than for targets in Color 2, $F(1,11)=$ 9.64, $p<.01, \eta_{\mathrm{p}}{ }^{2}=.47$. The overall RTs decreased as the experiment progressed, producing a main effect of block, $F(11,121)=4.07, p<.001, \eta_{\mathrm{p}}{ }^{2}=.27$. No significant interaction between target's color and block was observed, $F<1$.

To examine whether participants had acquired long-lasting changes in relative attentional priority for the two colors, we administered the testing phase, in which the target was equally likely to be in either color. A two-way ANOVA on target's color (Color 1 or Color 2) and block (Blocks 13-16) showed no difference in RTs between Color 1 and Color $2, F<1$ for the main effect of target's color. The interaction between target's color and block was also not significant, $F<1$. A followup $t$ test showed that the RT advantage for targets associated with Color 1 disappeared immediately in the testing phase: In the first testing block (Block 13), the RTs were comparable between Color 1 and Color 2, $t<1$.

\section{Discussion}

Experiment 1 both replicated and extended previous research on the target frequency effect in visual search. First, the training phase showed that participants were highly sensitive to the frequency of an incidental feature. Participants performed an orientation search task and reported the target's length, yet they were sensitive to the target's color frequency. Thus, the target frequency effect is not restricted to task-relevant features. Second, extending Kruijne and Meeter's (2015) findings, the frequency effect was short-lived; as soon as the target became equally likely to be in either color, the RT advantage for the previously high-frequency color disappeared. Thus, stable differences in the target's color frequency were insufficient to yield durable attentional biases. These data are consistent with both the episodic-retrieval account (Kruijne \& Meeter, 2015, 2016) and the diagnostic-value account. The 
Table 1 Percentage correct in all four experiments, shown separately for the training phase (first 12 blocks) and the testing phase (last four blocks of trials)

\begin{tabular}{llllll}
\hline & \multicolumn{2}{l}{ Training Phase } & & \multicolumn{2}{l}{ Testing Phase } \\
\cline { 2 - 3 } \cline { 6 - 7 } \cline { 5 - 6 } & Color 1 & Color 2 & & Color 1 & \multicolumn{1}{l}{ Color 2 } \\
\hline Experiment 1 & $97.4(0.5)$ & $96.2(0.6)^{* * *}$ & & $97.4(0.7)$ & $98.2(0.5)$ n.s. \\
Experiment 2 & $96.5(0.8)$ & $94.4(0.9)^{* *}$ & & $96.6(1.2)$ & $97.3(1.0)$ n.s. \\
Experiment 3 & $97.3(0.4)$ & $95.8(0.4)^{* * *}$ & & $94.3(1.0)$ & $94.6(0.6)$ n.s. \\
Experiment 4 & $96.5(0.6)$ & $94.3(0.9)^{* *}$ & & $97.4(0.3)$ & $97.3(0.5)$ n.s. \\
\hline
\end{tabular}

Color 1 was the high-frequency target color in the training phase. Standard errors of the means shown in parentheses. When comparing Color 1 and Color 2: n.s., not significant; ${ }^{* * *} p<.01{ }^{* * * *} p<.001$.

episodic-retrieval account accommodates these findings by postulating that in an orientaiton search task, color is not a searched-for feature and thus does not establish strong episodic-memory traces. The diagnostic-value account explains these findings in terms of the colors' diagnostic values in discriminating targets from distractors. Both colors always coincide with the target and never with a distractor. Therefore, they both receive maximal attentional priority. Short-term changes can still occur due to inter-trial priming of the target's colors, but long-term changes in relative attentional priority fail to emerge.

\section{Experiment 2}

Experiment 2 introduced a difference in diagnostic value between the two colors in addition to the different target's color

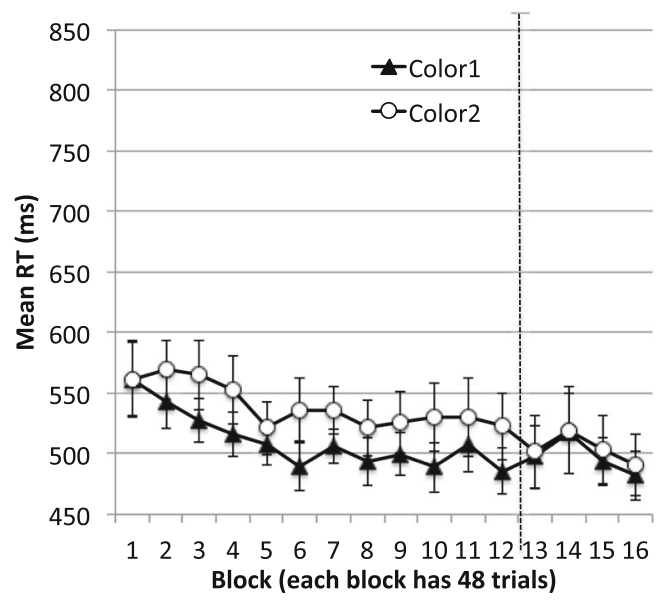

Fig. 2 Results from Experiment 1. When the target was more frequently in one color (Color 1) than in the other (Color 2) in the training phase (Blocks 1-12), participants were faster finding the target associated with the high-frequency color. This effect disappeared immediately in the testing phase (Blocks 13-16), in which the target was equally likely to be in either color. Error bars show \pm 1 standard error of the mean frequencies. Participants performed the same orientation search task and reported the target's length. Crucially, however, one color was more diagnostic of the item being a target rather than a distractor, relative to the other color. Specifically, during the training phase, $75 \%$ of the trials contained a target in Color 1 and a distractor in Color 2. The other $25 \%$ of the trials contained a target in Color 2 and a distractor in Color 1. Because the target was Color $175 \%$ of the time and Color 2 $25 \%$ of the time, its featural frequency, $P$ (feature $i \mid$ target), was the same as in Experiment 1. The crucial difference, though, was that Color 1 and Color 2 no longer had equal diagnostic values: Color 1 was now more diagnostic $[P($ target $\mid$ Color 1$)=.75]$ than Color $2[P($ target $\mid$ Color 2$)=$ $.25]$. If different diagnostic values support long-term changes in attention, this experiment should yield a persistent RT advantage for targets in Color 1 relative to Color 2. In contrast, the episodic-retrieval account predicts that long-term biases should not occur. This is because participants perform an orientation search task. The target's colors are not relevant and cannot yield strong episodic traces that support durable attentional biases.

\section{Method}

Participants Twelve naive participants (seven females, five males) with a mean age of 20.9 years completed Experiment 2.

Materials and stimuli Experiment 2 used the same ten colors as in Experiment 1. Two colors were assigned to be the colors of interest. They were drawn randomly from the set of four colors (red, green, yellow, and blue) and counterbalanced across participants. These will be referred to as Color 1 and Color 2. On each trial, the target was one color and one of the distractors was the other color. The other four distractors had colors randomly drawn from the remaining eight colors (Fig. 3). Other aspects of the experiment were the same as in Experiment 1.

Design Similar to Experiment 1, the experiment comprised a training phase and a testing phase. In the training phase, the target was in Color 1 on $75 \%$ of the trials. On those trials, one distractor was in Color 2. On the other 25\% of the trials, the target was in Color 2 and one distractor was in Color 1. During the testing phase, $50 \%$ of the trials had a Color 1 target with a Color 2 distractor, and the other $50 \%$ of the trials had a Color 2 target with a Color 1 distractor.

\section{Results}

In the training phase, an ANOVA on target's color and block showed that RTs were significantly faster when the target was in Color 1 rather than Color 2, $F(1,11)=11.21, p<.01, \eta_{\mathrm{p}}{ }^{2}=$ 


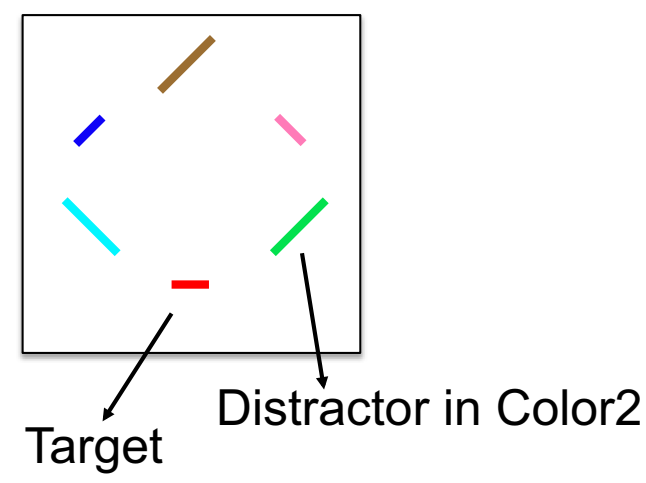

Fig. 3 Schematic illustration of a display used in Experiment 2. Participants searched for a target in a canonical (horizontal/vertical) orientation among tilted lines and reported the length of the target. The target was in Color 1 (e.g., red) on $75 \%$ of the trials, and in Color 2 (e.g., green) on $25 \%$ of the trials. When the target was in one color (e.g., Color 1 ), one of the distractors was in the other color (e.g., Color 2)

.51 , for the main effect of target's color (Fig. 4 Blocks 1-12). Overall RTs decreased as the experiment progressed, $F(11$, 121) $=8.20, p<.001, \eta_{\mathrm{p}}{ }^{2}=.43$ for the main effect of block. No significant interaction between target's color and block was observed, $F<1$.

Was the attentional bias for Color 1 durable? In the testing phase, the two colors were equal in both the target's color frequencies and diagnostic values. However, an ANOVA on target's color and block showed that participants continued to exhibit faster RTs for targets in Color 1 than those in Color 2, $F(1,11)=9.75, p<.01, \eta_{\mathrm{p}}{ }^{2}=.47$ for the main effect of target's color (Fig. 4 Blocks 13-16). This effect persisted for the entire testing phase, revealing no significant interaction between target's color and testing block, $F<1$.

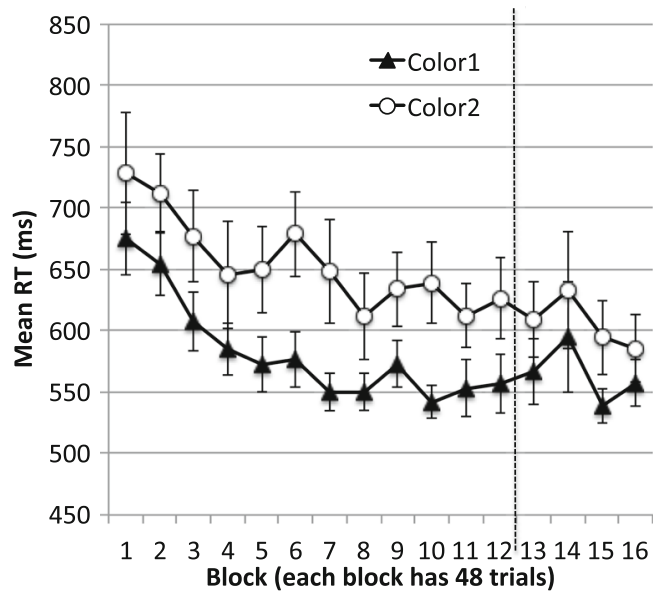

Fig. 4 Results from Experiment 2. In the training phase (Blocks 1-12), the target was three times as likely to be in Color 1 as in Color 2. In addition, Color 1 was more often associated with targets than with distractors, whereas the reverse was true for Color 2. Participants were faster finding targets in Color 1 than those in Color 2, and this effect persisted in the neutral testing phase (Blocks 13-16). Error bars show \pm 1 standard error of the mean

\section{Discussion}

In the first two experiments we manipulated two types of color-target association to examine their impact on attention. Using the same orientation search task, we observed both short-term and long-term changes in attention to a color that frequently coincided with the target. In the training phases of both experiments, the target's featural frequency favored Color 1: The target was more often associated with Color 1 than with Color 2. Participants were faster finding the target when it was in Color 1 rather than Color 2. However, the RT advantage dissipated in the neutral phase of Experiment 1. Thus, the target's featural frequency by itself induced only short-term changes in attention.

Experiment 2 differed from Experiment 1 in that not only was the target more often in Color 1 (rather than Color 2), but Color 1 also coincided more often with targets than with distractors. In other words, Color 1 was more diagnostic than Color 2 of a stimulus's status as a target rather than a distractor. The results showed that participants were sensitive to differences between the two colors' diagnostic values. To further confirm this finding, we tested for a cross-experiment interaction between target's color and experiments. First, with experiment (Exp. 1 vs. Exp. 2) as a between-subjects factor and target's color and block as within-subjects factors, a threeway ANOVA showed that the RT advantage for Color 1 over Color 2 was marginally greater in the training phase in Experiment 2 than in Experiment $1, F(1,22)=3.41, p<.08$, $\eta_{\mathrm{p}}{ }^{2}=.13$. This finding suggests that the training phase includes an additional learning component in Experiment 2. The marginal significance reported here may have resulted from a lack of power ( $N=12$ in each experiment). We validated this finding in later experiments. Second, the search advantage for Color 1 persisted in the neutral phase of Experiment 2 but disappeared in the neutral phase of Experiment 1, shown by a significant interaction between target's color and experiment in the testing phases, $F(1,22)=$ $5.29, p<.05, \eta_{\mathrm{p}}{ }^{2}=.31$. This finding indicates that a long-term attentional-learning component was acquired in Experiment 2 but not in Experiment 1. The comparisons between Experiments 1 and 2 are, of course, between experiments. The interaction effect should be validated in future studies using between-subjects, rather than between-experiments, designs.

\section{Experiment 3}

So far we have shown that differences in features' diagnostic values are important for durable changes in attention. However, it is unclear whether diagnostic values affect learning or the expression of learning. The diagnosticvalue account implies that differences in features' 
diagnostic values are necessary for inducing durable attentional biases toward the more diagnostic color. That is, the locus of the effect lies in the training phase. However, our data are also compatible with an alternative possibility: Presenting one color as a target and the other color as a distractor is a more sensitive test for long-term changes in attention. According to this account, it is possible that the training phases of both Experiments 1 and 2 induced longterm attentional biases toward Color 1, but the bias went undetected in the testing phase of Experiment 1. We will refer to this as the latent-learning account.

Latent learning has precedence in recent research on attention. Chelazzi et al. (2014) trained participants to search for a target that appeared in one of eight locations. Participants received high, medium, or low reward for different target locations. This training had no effect on the subsequent nonrewarded search when the display contained a single target. Latent learning nonetheless occurred and was detected when the display contained two targets. In this case, participants were more accurate in reporting the target in the previously highly rewarded location. Chelazzi et al. explained the difference between one-target and two-target trials in terms of the relative contributions of reward. On one-target trials the bottom-up signal of the target was strong, rendering reward-learning trivial. On two-target trials the bottomup signal was insufficient for resolving unique target detection, which heightened the impact of previous reward.

In Experiment 1, latent learning of Color 1 over Color 2 may have occurred but gone undetected in the testing phase. If this were the case, then an RT advantage for Color 1 might be revealed when the testing phase used the two-color displays of Experiment 2. To test this possibility, in Experiment 3 we used the same training procedure as in Experiment 1, but the same testing procedure as in Experiment 2.

\section{Method}

Participants Twenty-four participants ( 13 females, 11 males) with a mean age of 20.3 years completed Experiment 3 . The sample size was doubled relative to the first two experiments because the diagnostic-value account predicted a null effect.

Design As in Experiments 1 and 2, this experiment included 16 blocks of trials, with 48 trials per block. The first 12 blocks were the training phase. This phase was identical to the training phase of Experiment 1. The target was in Color $175 \%$ of the time and Color $225 \%$ of the time. Colors 1 and 2 never appeared as distractors. The last four blocks were the testing phase, which was identical to that phase of Experiment 2. Each trial contained both Color 1 and Color 2, one of which was a feature of the target, and

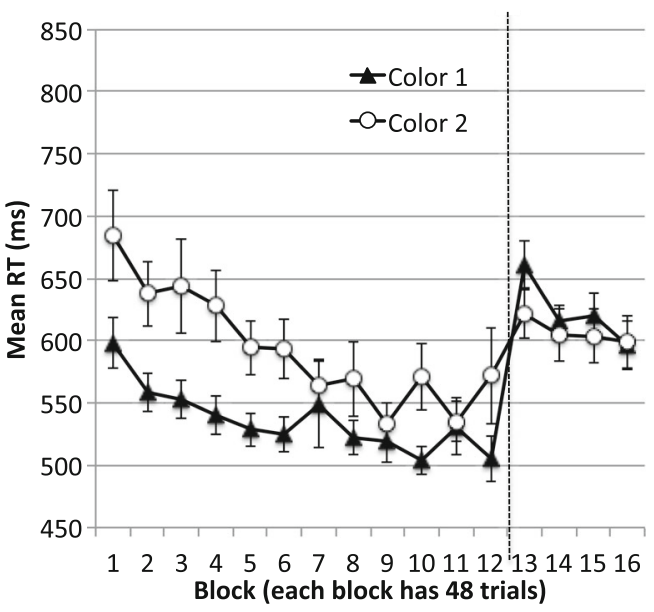

Fig. 5 Results from Experiment 3. Color 1 was the high-frequency color in Blocks 1-12, and Color 2 was the low-frequency color in Blocks 1-12. In Blocks 13-16, the two colors appeared simultaneously on each trial and were associated with the target with equal frequencies. The lines in Blocks 13-16 correspond to trials in which the target was in that color. Error bars show \pm 1 standard error of the mean

the other a feature of a distractor. We examined whether Experiment 1's training procedure induced durable changes in attention, when assessed using Experiment 2's testing procedure.

\section{Results}

Replicating Experiment 1, a two-way ANOVA on target's color and block showed that during the training phase, participants were significantly faster responding to Color 1 targets than to Color 2 targets, $F(1,23)=22.81, p<.001, \eta_{\mathrm{p}}{ }^{2}=$ .50 (Fig. 5 Blocks 1-12). In addition, RTs were faster in later than in earlier blocks, shown by a significant main effect of block, $F(11,253)=5.87, p<.001, \eta_{\mathrm{p}}^{2}=.20$. The target's frequency effect diminished across blocks, $F(11,253)=$ $1.91, p<.05, \eta_{\mathrm{p}}{ }^{2}=.08$, for the interaction between target's color and block.

Even though the testing phase presented both Color 1 and Color 2 concurrently, we found no evidence of long-term attentional biases (Fig. 5 Blocks 13-16). An ANOVA on target's color and block revealed no significant main effect of the target's color, $F(1,23)=1.39, p>.25$, nor an interaction between the target's color and block, $F(3,69)=1.55, p>.20$.

The lack of long-term attentional biases in Experiment 3 can be contrasted with that in Experiment 2. These two experiments were identical in the testing phase but differed in the nature of the training phase. An ANOVA on target's color and experiment (Exp. 2 vs. Exp. 3) revealed a significant interaction between target's color and experiment in the testing phase, $F(1,34)=7.08, p<.05, \eta_{\mathrm{p}}{ }^{2}=.17$. This comparison suggests that long-term effects are present in Experiment 2 but not in Experiment 3. 


\section{Discussion}

Experiment 3 used a potentially more sensitive measure of attention in the testing phase. On every trial the display contained both Color 1 and Color 2, one of which was the target and the other was a distractor. If participants had acquired a persistent attentional bias for Color 1, they should have faster RT when Color 1 was the search target and Color 2 was a distractor. In addition, they should be slower when a distractor was Color 1 , which could potentially capture attention away from the target. However, Experiment 3 showed no evidence of long-term attentional biases following Experiment 1's training procedure. RTs were comparable for Color 1 targets and Color 2 targets. This was the case even though the simultaneous presentation of Color 1 and Color 2 slowed down overall RTs. In fact, a two-way ANOVA on target's color and block (Block 12 vs. Block 13) showed slower RTs in the first block of the testing phase than in the last block of the training phase, $F(1,23)=17.03, p<.001, \eta_{\mathrm{p}}{ }^{2}$ $=.43$ for the main effect of block. This slowing suggests that Colors 1 and 2 competed for attention, making this paradigm a potentially more sensitive test for any residual attentional biases for one of the colors. Nonetheless, this procedure did not uncover any durable changes in relative attentional priority.

\section{Experiment 4}

Experiment 4 provided a complementary demonstration that differences in features' diagnostic values can yield long-term changes in attention. To this end, Experiment 4 used Experiment 2's training procedure, but the testing phase contained a random mix of two types of trials. In one type, the target was either Color 1 or Color 2, and the other color did not appear on the display. This was similar to Experiment 1's setup ("one-color" testing), a potentially less sensitive assessment of featural attentional biases. In another type, both colors appeared on the display, one coinciding with the target and the other with a distractor. This was similar to Experiment 2's setup ("two-color" testing). The diagnostic-value account predicts that participants should prioritize the previously highfrequency (and also more diagnostic) color, regardless of how the testing was conducted.

\section{Method}

Participants Twenty-four participants (19 females, five males) with a mean age of 19.7 years completed Experiment 4.

Design The first 12 blocks made up the training phase, which was identical to that of Experiment 2. The last four blocks

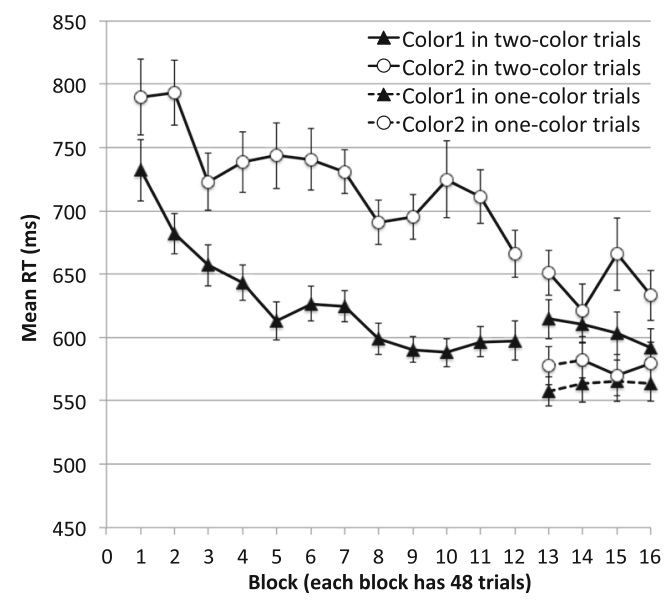

Fig. 6 Results from Experiment 4. In the training phase (Blocks 1-12), all trials were two-color trials (solid lines). In the testing phase, half of the trials were one-color trials (dashed lines), and the other half of the trials were two-color trials. Color 1 refers to the high-frequency (and also more diagnostic) color in the training phase. Error bars show \pm 1 standard error of the mean

made up the testing phase, which contained a mixture of displays. Half of the trials were similar to those of Experiment 1. On these trials, the target was either Color 1 or Color 2, and the other color was not present ("one-color" trials). The other half of the trials were similar to those of Experiment 2: Color 1 and Color 2 appeared simultaneously on each trial, one coinciding with the target and the other coinciding with a distractor ("two-color" trials). Overall, $25 \%$ of the trials within each block had a Color 1 target without Color 2 being presented, $25 \%$ of the trials had a Color 2 target without Color 1 being presented, $25 \%$ of the trials had a Color 1 target with one Color 2 distractor, and another $25 \%$ of the trials had a Color 2 target with one Color 1 distractor. All trials were randomly mixed within each block.

\section{Results}

Replicating Experiment 2, a two-way ANOVA on target's color and block showed that during the training phase, participants were significantly faster responding to targets in Color 1 than to those in Color 2, $F(1,23)=36.09, p<.001, \eta_{\mathrm{p}}{ }^{2}=.61$ (Fig. 6, Blocks 1-12). RTs were faster in later than in earlier blocks, $F(11,253)=13.44, p<.001, \eta_{\mathrm{p}}{ }^{2}=.37$. The effect of target color was stronger in later blocks, resulting in a significant interaction between target's color and block, $F(11,253)$ $=2.12, p<.05, \eta_{\mathrm{p}}{ }^{2}=.08$. Replicating Experiments 1 and 2, the training effect was greater in Experiment 4 than in Experiment $3, F(1,46)=4.23, p<.05, \eta_{\mathrm{p}}{ }^{2}=.09$ for the interaction between target's color and experiment.

Figure 6 (Blocks 13-16) plots RTs separately for the onecolor and two-color trials. Long-term attentional biases are apparent from these data. A three-way ANOVA on trial type 
(one-color vs. two-color), target's color (Color 1 vs. Color 2), and block (13-16) showed a significant main effect of trial type. RTs were faster on one-color than on two-color trials, $F(1,23)=87.61, p<.001, \eta_{\mathrm{p}}{ }^{2}=.79$, consistent with stronger competition between the two colors when they were presented together. Importantly, participants were faster responding to targets in Color 1 than to those in Color 2, $F(1,23)=9.79, p<$ $.01, \eta_{\mathrm{p}}{ }^{2}=.30$, yielding a significant main effect of target's color. All other factors failed to reach significance, largest $F(1,23)=2.49, p>.12$. Two follow-up ANOVAs were conducted, separately for one-color and two-color trials, using target's color and block as factors. The main effects of target's color was significant in both one-color testing trials, $F(1,23)=$ $7.72, p<.02, \eta_{\mathrm{p}}{ }^{2}=.25$, and two-color testing trials, $F(1,23)=$ $6.51, p<.02, \eta_{\mathrm{p}}{ }^{2}=.22$.

Together, Experiments 3 and 4 showed that the training procedure, rather than the testing procedure, determined whether long-term changes in attention would be revealed. These data support the idea that whereas differences in target's featural frequency can induce shortterm changes, how diagnostic some features are for distinguishing targets from distractors can additionally yield long-term changes in attention.

\section{Repetition priming}

The diagnostic-value account acknowledges the presence of short-term priming when the target's features repeat. Here we examined repetition priming for target color across consecutive trials in the training phase. The data were combined for Experiments 1 and 3 and for Experiments 2 and 4. Trials were coded on the basis of whether the target's color was Color 1 or Color 2, and whether it had the same color as the preceding trial's target. RTs were significantly faster on color repeat trials than on color switch trials, $p \mathrm{~s}<.01$. We then examined whether the repetition priming was comparable between the two colors. To adjust for differences in baseline RTs across experiments, priming was expressed as the percent RT saving, computed as [(switch_RT - repeat_RT)/mean_RT]. Priming was then analyzed in an ANOVA on training procedure (Exps. $1 \& 3$ vs. Exps. 2\&4) and the target's color (Color 1 or Color 2). Priming was comparable between Color 1 and Color 2 and between the two types of training procedures, resulting in the lack of main effects or an interaction (smallest $p=.23$ ). These data suggest that repetition priming contributed to similar degrees under the two types of training procedures used in this study. Table 2 displays the mean RT and priming results.

\section{Explicit recognition}

In all experiments, about half of the participants both thought that the two colors appeared with unequal frequencies, and were able to correctly identify the high-frequency color. These
Table 2 Mean reaction times (RTs) and percent RT savings in the training phase, shown separately for Experiments 1 and 3 and Experiments 2 and 4 (SEs in parentheses)

\begin{tabular}{cllll}
\hline & $\begin{array}{l}\text { Target's } \\
\text { Color }\end{array}$ & $\begin{array}{l}\text { Mean RT } \\
(\mathrm{ms})\end{array}$ & \multirow{2}{*}{$\begin{array}{l}\text { Percent } \\
\text { RT Saving (\%) }\end{array}$} \\
\cline { 3 - 4 } & & $\begin{array}{l}\text { Color } \\
\text { Repeat }\end{array}$ & $\begin{array}{l}\text { Color } \\
\text { Switch }\end{array}$ \\
\hline Exps. & Color 1 & $518(11)$ & $541(13)$ & $4.3(0.9)$ \\
$1 \& 3$ & Color 2 & $561(15)$ & $576(16)$ & $2.0(2.2)$ \\
Exps. & Color 1 & $601(10)$ & $630(11)$ & $4.9(0.9)$ \\
$2 \& 4$ & Color 2 & $679(15)$ & $710(17)$ & $4.1(1.1)$ \\
\hline
\end{tabular}

were the "aware" participants, making up 52\% of the participants across the four experiments (33\% in Exp. 1, 50\% in Exp. 2, 63\% in Exp. 3, and 50\% in Exp. 4). Still, a substantial proportion of the participants thought that the two target colors occurred equally often $(32 \%)$ or assigned a higher percentage to the low-frequency target $(16 \%)$. These were the "unaware" participants (a total of 48\%). To examine whether explicit awareness influenced the search results, we reran the analyses reported in this study with awareness group added as a between-subjects factor. In none of the experiments did awareness group interact with the other experimental factors, $F \mathrm{~s}<1$. (Analysis details can be found at http://jianglab.psych.umn.edu/ FeatureProbability/ShortLongTermLearningAwareness.pdf.)

To attain higher statistical power, we combined the data from Experiments 1 and 3 and the data from Experiments 2 and 4. In Experiments 1 and 3, neither the aware $(N=19)$ nor the unaware $(N=17)$ participants showed RT differences for Color 1 and Color 2 in the testing phase, $F \mathrm{~s}<1$. In Experiments 2 and 4, both the aware $(N=18)$ and the unaware $(N=18)$ participants showed faster RTs to Color 1 than to Color 2 in the testing phase, $p s<.02$. Thus, the main conclusion reached in this study held for both aware and unaware participants. Consistent with Kruijne and Meeter (2016), frequency effects on visual search did not depend on explicit awareness.

\section{General discussion}

Intuitively, features frequently associated with a visual search target should be prioritized. This intuition was supported by our training-phase data. Across all four experiments, a color that frequently coincided with the search target was associated with faster search RTs. The target's frequency effect was found even though color was, strictly speaking, taskirrelevant; orientation was the target's defining property, and length was the target's reported property. The training-phase 
data suggest that target's featural frequency affects attentional allocation (Hasher \& Zacks, 1984).

This study provides important insights into the conditions under which feature-target associations lead to either long- or short-term attentional biases. We showed in Experiments 1 and 3 that attentional priority for the previously high-frequency target diminished rapidly in a neutral testing phase. This finding suggests that the frequency effect observed in these experiments is likely supported by transient mechanisms, such as inter-trial priming of the search target. The more frequent target color also repeates more often across trials, accumulating greater inter-trial priming in the training phase. Because intertrial priming dissipates rapidly (Maljkovic \& Nakayama, 2000), it does not support sustained attentional biases when the two target colors become equally frequent. In fact, Sha et al. (2017) estimated inter-trial priming over eight successive trials and found that this effect could fully explain the RT advantage for the more frequent target color.

The second contribution of this study is the empirical demonstration in Experiments 2 and 4 that the differences in the diagnostic values of features for discriminating targets from nontargets produce long-term statistical learning. Durable target frequency effects have been reported previously in colorshape conjunction search tasks, but not in color singleton search tasks (Kruijne \& Meeter, 2015). The present study extends this finding to feature search tasks, and it is important especially because the finding is unexpected from previous theoretical work. According to Kruijne and Meeter (2015), durable frequency effects are found when people maintain specific target's defining features during search, but not when people accomplish search by detecting local stimulus differences. This account is consistent with the data showing differences between Kruijne and Meeter's (2015) conjunction and singleton search tasks. However, it does not adequately explain why the feature search task used in the present study yielded only transient changes in Experiments 1 and 3, but durable changes in Experiments 2 and 4. In all four experiments, participants searched for vertical or horizontal lines, so color was not part of the search template. According to the episodic-retrieval account, Color 1 or Color 2 should not have left episodic traces strong enough to support long-term changes in attention. One might argue that owing to the correlational structure between color and target, participants might have actively searched for Color 1 and Color 2. If this were the case, we should have observed long-term attentional biases in all experiments (especially in Exps. 1 and 3, in which the correlational structure was strong), not just in Experiments 2 and 4. Our study suggests that the time scale of the target frequency effect is modulated by factors other than the accumulation of episodic traces.

A third contribution of the present study is that we provided initial evidence for a theoretical distinction between two types of feature-target association. Both types are expressed in terms of conditional probability, but they have potentially different functional roles. The target's featural frequency, expressed as $P$ (feature $i \mid$ target), indexes how frequently a feature $i$ target occurs. In contrast, a feature's diagnostic value, expressed as $P($ target $\mid$ feature $i)$, indexes feature $i$ 's usefulness in visual search. A color with higher diagnostic value is more predictive of the target than of distractors and can facilitate visual search. This may explain why we observed durable attentional biases in Experiments 2 and 4. One way to understand this proposal is by considering attention as a mechanism that biases competition toward diagnostic features (Desimone \& Duncan, 1995). In visual search, the crucial competition is between targets and distractors, rather than among the targets themselves. When two features are both maximally indicative of targets, they can both be prioritized relative to nontarget features. But when a feature more often coincides with a target and another feature more often coincides with a distractor, these features compete for attentional priority, and the more diagnostic feature wins.

This diagnostic-value account is consistent with a recent study examining the role of statistical learning on attentional capture. Using the contingent capture paradigm (Folk, Remington, \& Johnston, 1992), Cosman and Vecera (2014) had participants respond to the identity of a red or green target presented along with a red or green distractor. On $80 \%$ of trials the target was in one color (e.g., red), and on $20 \%$ of trials in the other color. Targets were preceded by a red or green cue at one of the locations. According to our analysis, the use of both colors as both targets and distractors established a higher diagnostic value for the high-frequency target color. Indeed, in a subsequent neutral testing phase with equal color-target assignments, Cosman and Vecera found stronger capture of attention when the cue was in the high-frequency color than when it was in the low-frequency color. Unlike in the present study, Cosman and Vecera did not examine conditions under which such effects were transient or durable, nor did they formulate a general account of the effects of stable frequency statistics on attention.

Our study supports the idea that humans are sensitive to conditional probabilities in visual input (Orbán, Fiser, Aslin, \& Lengyel, 2008). More broadly, conditional probabilities are key elements in Bayesian inference in vision, reasoning, and language (Kalish, Griffiths, \& Lewandowsky, 2007; Kersten, Mamassian, \& Yuille, 2004). The data presented here demonstrate that such statistical information is also extracted to support visual search. As is the case in standard Bayesian computation, the two conditional probability terms, commonly given as $P(\mathrm{~A} \mid \mathrm{B})$ and $P(\mathrm{~B} \mid \mathrm{A})$, correspond to different concepts and serve different functional roles. Our study suggests that these functional differences may support learning at multiple time scales.

At first glance, the diagnostic-value account appears to be inconsistent with one finding in the literature: the lack of 
durable frequency effects in singleton search tasks. Consider the task of finding an odd-colored target (e.g., red) among homogeneous colored distractors (e.g., green; Kruijne \& Meeter, 2015). When most trials are red among green and few are green among red, red is more often a target than a distractor, and green is more often a distractor than a target. Why does this difference in diagnostic value not induce a persisting attentional bias for red? We believe that the reason is that when people search for a color singleton, they primarily adopt the singleton-search mode (Bacon \& Egeth, 1994), such as detecting a local discontinuity in color. The adoption of this mode likely interferes with statistical learning of the specific target color. How visual statistics are computed for singleton search remains to be tested.

Our experiments, along with that of Cosman and Vecera (2014), establish the importance of diagnostic value in longterm statistical learning. They do not, however, specify the nature of this learning. We have proposed that frequency information accrues from trial to trial, with the resulting conditional probabilities being computed independently and in parallel for all target and distractor colors. Another possibility is that the simultaneous presence of two potential target colors leads to trial-by-trial inhibition of the color associated with the distractor. Attentional priority would then be based on a computation of accumulated strength across trials. Existing studies do not provide a definitive answer as to the nature of the underlying learning.

\section{Conclusions}

In this study we examined the roles of featural frequency in inducing short-term and long-lasting changes in attention. We found that both short-term and long-term mechanisms contribute to people's sensitivity to featural frequency in visual search. Our results support the claim that short-term changes operate on the target's featural frequency (e.g., inter-trial priming), whereas long-term changes may depend on the ratio of a feature coinciding with the target rather than a distractor (its diagnostic value). We propose that the diagnostic value of a feature in discriminating targets from distractors is important for inducing durable changes in attention.

Author note We thank Wilma Koutstaal for discussion, Doug Addleman and Nikita Salovich for editing, Martijn Meeter for comments on an earlier draft of the article, and Xinyi Wang, Yang Yang, Hyejin Lee, Michaela DeBolt, and Gracelynn Goh for help with data collection.

\section{References}

Bacon, W. F., \& Egeth, H. E. (1994). Overriding stimulus-driven attentional capture. Perception \& Psychophysics, 55, 485-496. doi:10. 3758/BF03205306
Brainard, D. H. (1997). The psychophysics toolbox. Spatial Vision, 10, 433-436. doi:10.1163/156856897X00357

Chelazzi, L., Eštočinová, J., Calletti, R., Gerfo, E. L., Sani, I., Della Libera, C., \& Santandrea, E. (2014). Altering spatial priority maps via reward-based learning. Journal of Neuroscience, 34, 85948604. doi:10.1523/JNEUROSCI.0277-14.2014

Chun, M. M., \& Jiang, Y. (2003). Implicit, long-term spatial contextual memory. Journal of Experimental Psychology: Learning, Memory, and Cognition, 29, 224-234. doi:10.1037/0278-7393.29.2.224

Cosman, J. D., \& Vecera, S. P. (2014). Establishment of an attentional set via statistical learning. Journal of Experimental Psychology: Human Perception and Performance, 40, 1-6. doi:10.1037/a0034489

Desimone, R., \& Duncan, J. (1995). Neural mechanisms of selective visual attention. Annual Review of Neuroscience, 18, 193-222. doi:10.1146/annurev.ne.18.030195.001205

Folk, C. L., Remington, R. W., \& Johnston, J. C. (1992). Involuntary covert orienting is contingent on attentional control settings. Journal of Experimental Psychology: Human Perception and Performance, 18, 1030-1044. doi:10.1037/0096-1523.18.4.1030

Gebhart, A. L., Aslin, R. N., \& Newport, E. L. (2009). Changing structures in midstream: Learning along the statistical garden path. Cognitive Science, 33, 1087-1116. doi:10.1111/j.1551-6709.2009. 01041.x

Godwin, H. J., Menneer, T., Cave, K. R., Helman, S., Way, R. L., \& Donnelly, N. (2010). The impact of relative prevalence on dualtarget search for threat items from airport X-ray screening. Acta Psychologica, 134, 79-84. doi:10.1016/j.actpsy.2009.12.009

Godwin, H. J., Menneer, T., Riggs, C. A., Cave, K. R., \& Donnelly, N. (2015). Perceptual failures in the selection and identification of lowprevalence targets in relative prevalence visual search. Attention, Perception, \& Psychophysics, 77, 150-159. doi:10.3758/s13414014-0762-8

Hasher, L., \& Zacks, R. T. (1984). Automatic processing of fundamental information: The case of frequency of occurrence. American Psychologist, 39, 1372-1388. doi:10.1037/0003-066X.39.12.1372

Hout, M. C., Walenchok, S. C., Goldinger, S. D., \& Wolfe, J. M. (2015). Failures of perception in the low-prevalence effect: Evidence from active and passive visual search. Journal of Experimental Psychology: Human Perception and Performance, 41, 977-994. doi:10.1037/xhp0000053

Jiang, Y. V., Sha, L. Z., \& Remington, R. W. (2015). Modulation of spatial attention by goals, statistical learning, and monetary reward. Attention, Perception, \& Psychophysics, 77, 2189-2206. doi:10. 3758/s13414-015-0952-Z

Jiang, Y. V., Swallow, K. M., Rosenbaum, G. M., \& Herzig, C. (2013). Rapid acquisition but slow extinction of an attentional bias in space. Journal of Experimental Psychology: Human Perception and Performance, 39, 87-99. doi:10.1037/a0027611

Jungé, J. A., Scholl, B. J., \& Chun, M. M. (2007). How is spatial context learning integrated over signal versus noise? A primacy effect in contextual cueing. Visual Cognition, 15, 1-11. doi:10.1080/ 13506280600859706

Kalish, M. L., Griffiths, T. L., \& Lewandowsky, S. (2007). Iterated learning: Intergenerational knowledge transmission reveals inductive biases. Psychonomic Bulletin \& Review, 14, 288-294. doi:10. 3758/BF03194066

Kersten, D., Mamassian, P., \& Yuille, A. (2004). Object perception as Bayesian inference. Annual Review of Psychology, 55, 271-304. doi:10.1146/annurev.psych.55.090902.142005

Kruijne, W., \& Meeter, M. (2015). The long and the short of priming in visual search. Attention, Perception, \& Psychophysics, 77, 15581573. doi:10.3758/s13414-015-0860-2

Kruijne, W., \& Meeter, M. (2016). Long-term priming of visual search prevails against the passage of time and counteracting instructions. Journal of Experimental Psychology: Learning, Memory, and Cognition, 42, 1293-1303. doi:10.1037/xlm0000233 
Maljkovic, V., \& Nakayama, K. (1994). Priming of pop-out: I. Role of features. Memory \& Cognition, 22, 657-672. doi:10.3758/ BF03209251

Maljkovic, V., \& Nakayama, K. (2000). Priming of popout: III. A shortterm implicit memory system beneficial for rapid target selection. Visual Cognition, 7, 571-595. doi:10.1080/135062800407202

Orbán, G., Fiser, J., Aslin, R. N., \& Lengyel, M. (2008). Bayesian learning of visual chunks by human observers. Proceedings of the National Academy of Sciences, 105, 2745-2750. doi:10.1073/ pnas.0708424105

Pelli, D. G. (1997). The VideoToolbox software for visual psychophysics: Transforming numbers into movies. Spatial Vision, 10, 437-442. doi:10.1163/156856897X00366
Sha, L. Z., Remington, R. W., \& Jiang, Y. V. (2017). Rapid and selective updating of the target template in visual search. Journal of Vision, 17(1), 36. doi:10.1167/17.1.36

Walthew, C., \& Gilchrist, I. D. (2006). Target location probability effects in visual search: An effect of sequential dependencies. Journal of Experimental Psychology: Human Perception and Performance, 32, 1294-1301. doi:10.1037/0096-1523.32.5.1294

Yu, R. Q., \& Zhao, J. (2015). The persistence of the attentional bias to regularities in a changing environment. Attention, Perception, \& Psychophysics, 77, 2217-2228. doi:10.3758/s13414-015-0930-5 